Écrire

l'histoire

\section{Écrire l'histoire}

Histoire, Littérature, Esthétique

17 | 2017

La petite histoire

\title{
Une « petite histoire » corse en 1925
}

Ou comment le Napoléon d'Abel Gance croise l'histoire du fascisme français

\section{Dimitri Vezyroglou}

\section{(2) OpenEdition \\ 12 Journals}

Édition électronique

URL : http://journals.openedition.org/elh/1215

DOI : $10.4000 /$ elh. 1215

ISSN : 2492-7457

Éditeur

CNRS Éditions

\section{Édition imprimée}

Date de publication : 5 septembre 2017

Pagination : 87-94

ISBN : 978-2-271-11657-4

ISSN : 1967-7499

\section{Référence électronique}

Dimitri Vezyroglou, «Une « petite histoire » corse en 1925 », Écrire l'histoire [En ligne], 17 | 2017, mis en ligne le 28 septembre 2020, consulté le 29 octobre 2020. URL : http://journals.openedition.org/elh/ 1215 ; DOl : https://doi.org/10.4000/elh.1215 


\section{Une "petite histoire " corse en 1925}

\section{Ou comment le Napoléon d'Äbel Gance croise l'histoire du fascisme français}

À sa présentation au printemps 1927, le film Napoléon réalisé par Abel Gance en 1925-1926 est accueilli de façon très contrastée. Si les exploits techniques et les envolées épico-lyriques qu'il contient suscitent un enthousiasme à peu près général, certains critiques ne manquent pas de relever, voire de dénoncer le portrait apologétique du chef qui vient dompter le désordre révolutionnaire (l'action du film se déroule essentiellement entre 1792 et 1796). Le plus virulent est sans doute Léon Moussinac, qui, dans sa chronique cinématographique de L'Humanité et malgré toute l'estime et l'amitié qu'il porte au réalisateur, vilipende un "Bonaparte pour apprentis fascistes ${ }^{1} »$ dont la représentation idolâtre est appuyée sur un dénigrement de la Révolution française, et brocarde sur un ton à la fois ironique et acerbe ce héros «heureusement survenu pour tout remettre en ordre selon les bonnes lois de la discipline, de l'autorité et de la patrie, pour tout dire: de la dictature militaire ${ }^{2} »$. Cette virulence du critique communiste s'explique aisément, dans un contexte où, aux frontières de la France, la dictature fasciste s'affirme et se durcit en Italie tandis que le parti nazi entreprend sa percée dans le corps électoral allemand, mais aussi où, en France même, les ligues d'extrême droite sont de plus en plus actives et influentes. Paru au lendemain du discours de Constantine, où le ministre de l'Intérieur radical Albert Sarraut s'était exclamé «Le communisme, voilà $l^{\prime}$ ennemi ${ }^{3}$ », lançant insi une vaste offensive des pouvoirs publics contre le mouvement ouvrier, l'article de Moussinac a bien l'allure d'une instrumentalisation de la critique cinématographique dans un réflexe d'autodéfense communiste.

Pourtant, à y regarder de plus près, l'histoire de la production du film de Gance contient des éléments qui font apparaître, au-delà de l'appréciation politique subjective d'une certaine critique au moment de sa sortie, une proximité objective sinon avec l'idéologie fasciste, tout au moins avec certains de ses acteurs et vecteurs; une proximité, qui plus est, avec laquelle le réalisateur lui-même a, jusqu'à un certain point, sciemment joué. Parmi ces éléments, une "petite histoire» corse concentre, 
sous des dehors inoffensifs de folklore politique local, les principaux enjeux de cette rencontre entre Napoléon et un certain fascisme français.

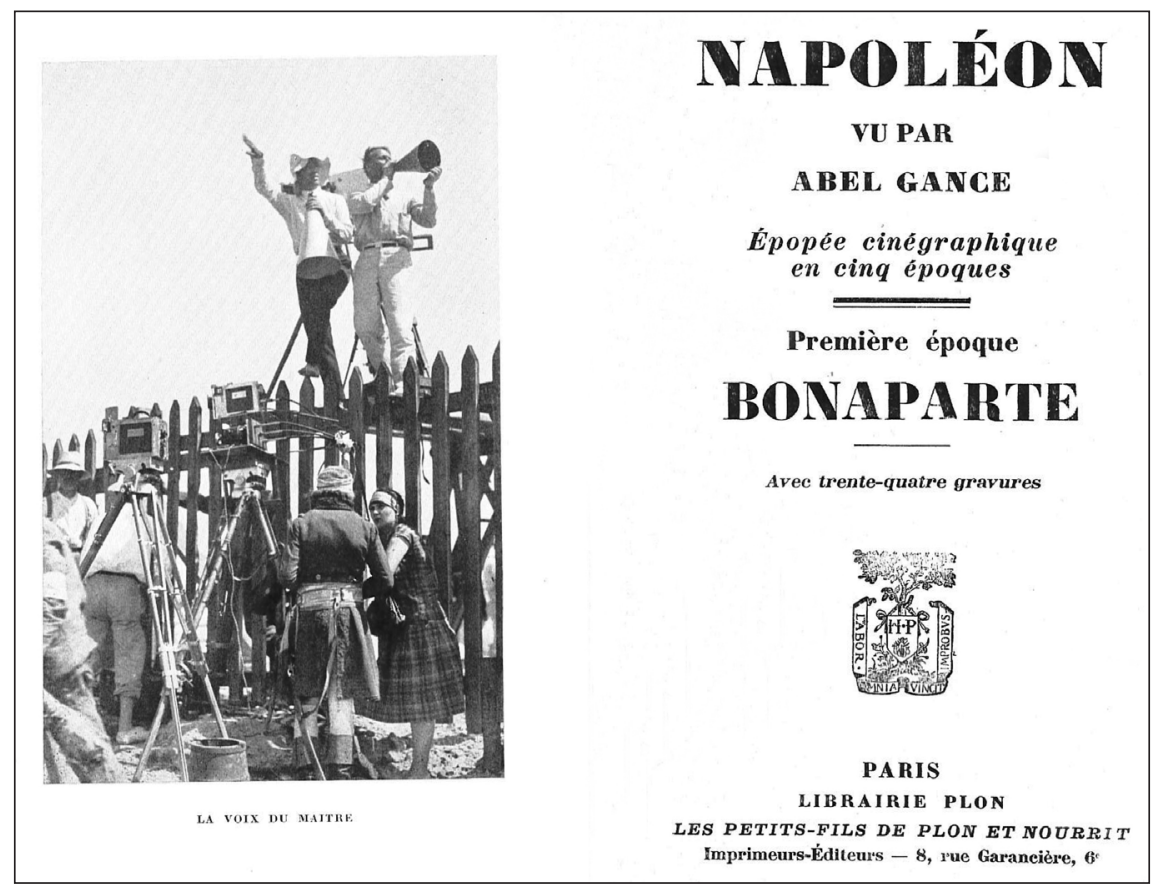

Abel Gance dirigeant une prise de vues dans le Var

\section{Bonaparte à Ajaccio: une tempête dans un verre d'eau?}

À son arrivée en Corse, au début d'avril 1925, pour le tournage de séquences situées en 1793 et mettant en scène un Bonaparte échouant à rallier son île natale à la République, l'équipe de tournage de Napoléon se trouve prise à partie dans le contexte effervescent des élections municipales qui, à Ajaccio comme sur l'ensemble du territoire métropolitain, doivent se dérouler les 3 et 10 mai. En 1919, le radical et républicain Jérôme Peri avait ravi la mairie aux bonapartistes qui la tenaient sans interruption depuis 1884, mettant ainsi fin au long règne de Dominique Pugliesi-Conti (1904-1919), artisan du ralliement du mouvement bonapartiste corse à la République, qui perd d'ailleurs aussi, toujours en 1919, son siège de député. Cette défaite avait causé une crise au sein du Comité central bonapartiste $(\mathrm{CCB})$, parti politique municipal conservateur se donnant pour objet de faire vivre le glorieux souvenir napoléonien. Tournant le dos au républicanisme de Pugliesi-Conti, le CCB se réoriente durant le mandat de Peri dans un sens de plus en plus nettement antirépublicain, sous la double influence de son nouveau chef, Dominique Paoli, et de son principal financeur, qui n'est autre que l'enfant du pays François Coty. Célèbre parfumeur, Corse de naissance 
- de son vrai nom Joseph Marie François Spoturno - et détenteur d'une immense fortune, par ailleurs directeur politique du Figaro, ce dernier est alors l'une des personnalités les plus influentes de l'extrême droite française ${ }^{4}$.

L'occasion était trop belle pour ces bonapartistes nouvelle manière, à quelques semaines des élections par lesquelles ce nouveau CCB compte bien refermer la parenthèse républicaine ouverte par Peri, de se saisir de la présence dans la ville impériale d'une troupe d'acteurs, de figurants et de techniciens occupés à faire revivre la glorieuse jeunesse de leur héros.

Voici comment le reporter Pierre Scize, qui couvre l'aventure gancienne pour Lectures pour tous, narre cet épisode périphérique du tournage:

C'est dans cette cité en proie au souvenir [de l'Empereur] qu'Abel Gance débarquait pour demander au maire en fonction les autorisations nécessaires pour «tourner» Napoléon. L'excellent homme leva les bras au ciel:

«En ce moment! Promener dans les rues d'Ajaccio un Bonaparte à cheval, en costume! Vous voulez ma mort! Les esprits sont déjà surexcités! Nous allons à une émeute! Repassez après les élections.»

Il fallut faire comprendre au digne magistrat municipal qu'on ne saurait renvoyer ainsi des artistes, des ingénieurs, des décorateurs, des costumiers, des perruquiers, des électriciens, des opérateurs, des régisseurs, des artificiers, un cuisinier, des dactylographes, un comptable, un secrétaire général, etc.

Alors - et parce que, bien que républicain, le maire était avant tout Ajaccien, et, comme tel, sensible au souvenir de l'Autre, on vit ce spectacle cornélien: il donna les autorisations nécessaires, fournissant ainsi des armes, un moyen de propagande au parti qui le combattait. Retenez le nom de ce maire: il s'appelait Jérôme Peri. Eustache de Saint-Pierre [l'un des bourgeois de Calais] fut plus courageux, mais non plus magnanime. Encore eut-il la vie sauve, tandis que le maire d'Ajaccio fut mis en minorité. Or, en Corse, on préfère la mort à la disgrâce. J'immole cette victime sur ton autel, ô Cinéma!

Le soir du même jour, le chef du parti bonapartiste demandait audience au secrétaire général du film.

«Donnez-nous votre Napoléon. Nous lui promettons une apothéose. Il défilera à cheval rue Fesch, couvert de fleurs par la population. Ce sera une minute inoubliable pour lui. Et pour vous, quelle publicité!»

La tentation était forte! Mais le préfet de la Corse, arbitre des partis en présence, avait demandé à la Compagnie Abel Gance de se tenir farouchement au-dessus de la mêlée. La rue Fesch dut se passer de l'Empereur. Elle n'en est pas encore consolée. ${ }^{5}$

Cette «petite histoire» - si petite, en réalité, qu'elle confine au nonévénement - est ici contée avec légèreté; elle apparaît sous un jour plus léger encore sous la plume de l'historien Kevin Brownlow, qui l'évoque lui aussi parmi les péripéties du tournage ${ }^{6}$. Elle est pourtant lourdement chargée de sens politique, et la présence, dans l'ombre, de Coty lui donne une dimension qui dépasse largement le contexte de la vie politique ajaccienne. En effet, on peut comprendre, tout d'abord, l'inquiétude 
de Peri devant ce qui est susceptible de devenir bien plus que le prétexte d'une ferveur folklorique: une arme de propagande en faveur d'ennemis politiques qui fondent leur combat sur la remise en cause de la République elle-même. Cette crainte est par ailleurs confortée par l'initiative de Paoli et du CCB sollicitant de la part de l'administration $\mathrm{du}$ film et de l'équipe de tournage un concours actif à une entreprise qui, si elle s'était concrétisée dans ce contexte, eût constitué une véritable provocation symbolique: faire défiler l'acteur Albert Dieudonné costumé en Bonaparte dans la rue du Cardinal-Fesch, principale artère de la vieille ville, qui débouche sur la zone où se trouvent à la fois la maison natale de Napoléon et l'hôtel de ville. Et ce même si, précisément, cette partie
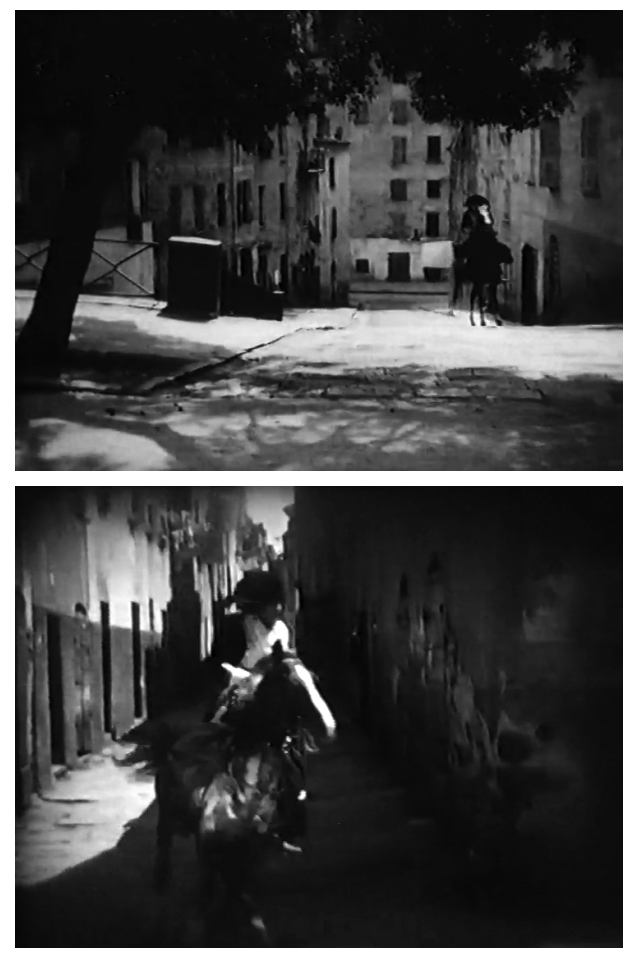

Bonaparte (Albert Dieudonné) galopant dans les rues d'Ajaccio $\mathrm{du}$ film de Gance montre un Bonaparte farouchement républicain et partisan du maintien de la Corse dans le giron français, affrontant Pascal Paoli, dont le souvenir vibre encore glorieusement dans les mémoires corses, et arrachant, pour le sauver, le drapeau tricolore à la façade dudit hôtel de ville.

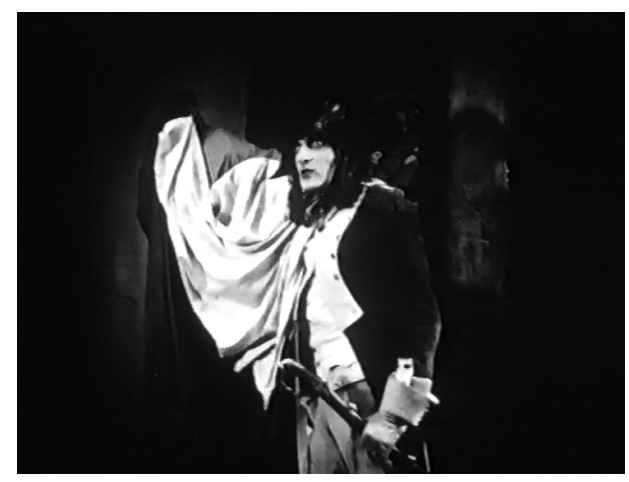

Bonaparte (Albert Dieudonné) défiant Pascal Paoli

Enfin, l'administration du film (il s'agit, semble-t-il, de Pierre Bonardi et Henri Andréani, et non de Gance luimême, qui n'était pas encore arrivé sur les lieux ${ }^{7}$ ) ne paraît pas avoir de prime abord reçu avec réticence cette proposition de Paoli, qui offrait, si l'on n'y regardait pas de trop près, l'occasion d'une publicité inédite et bienvenue, et ce n'est que grâce à l'arbitrage préfectoral que cette appropriation politique du film n'a pas eu lieu. Quoi qu'il en soit, Peri est défait lors des élections, Coty est élu conseiller municipal et Paoli maire, fonction à laquelle il sera reconduit jusqu'en 1943, en dehors de l'intervalle 1931-1934, où il cède précisément son siège à Coty. Durant ses mandats, Paoli se distinguera par des propos antirépublicains virulents et par une admiration explicite et ostensible pour le régime fasciste italien ${ }^{8}$. 
L'histoire de Napoléon croise donc celle $\mathrm{du}$ fascisme français, au sein d'un épisode resté anecdotique, mais qui aurait pu voir le film de Gance instrumentalisé par un mouvement dans lequel Coty est directement impliqué. Ce sont les institutions républicaines qui ont empêché cette instrumentalisation. Ce rendez-vous manqué n'est pourtant pas seulement un fait isolé, une «petite histoire» de tournage.

\section{De Coty à Mussolini : liaisons dangereuses}

C'est en effet quelques mois plus tard, au début de l'automne 1925, que l'affaire Coty éclate publiquement. Alors que la production du film est arrêtée depuis trois mois à la suite du décès de son principal financeur, l'industriel allemand Hugo Stinnes, et de la faillite de son entreprise, Coty signe dans son quotidien, sur deux colonnes à la une, un article motivant son refus de participer à la reprise $d u$ financement du film 9 . Ce refus vient contredire la tentative d'instrumentalisation que le propre parti de Coty avait entreprise cinq mois plus tôt à Ajaccio, alors même que celui qui est en train de s'affirmer comme l'un des principaux soutiens des ligues d'extrême droite aurait pu trouver son compte dans une superproduction exaltant la figure du chef militaire mettant fin aux troubles révolutionnaires.

L'argumentation du directeur politique du Figaro est simple:

Depuis la conclusion de la paix, et surtout en ce moment précis, tout l'effort de la propagande antifrançaise tend à montrer la France impérialiste, militariste, affamée de gloire et de conquêtes, consacrant ses ressources à de formidables armements, implacable dans sa haine contre la pauvre Allemagne, usurpant l'hégémonie sur le continent européen. ${ }^{10}$
Selon Coty, cette accusation, qui s'exprime particulièrement chez les anciens Alliés d'outre-Manche et d'outre-Atlantique, est soutenue par une propagande allemande systématique; un film français exaltant l'épopée napoléonienne ne peut donc que contribuer objectivement à cette propagande; cela est d'autant plus vrai que, quelle que puisse être la bonne foi de Gance, le fait que le nationaliste allemand Hugo Stinnes ait été le premier financeur du film en signale par ailleurs la destination subjectivement hostile à la France.

À la lumière du contexte, cette violente attaque de l'entreprise gancienne apparaît clairement comme un prétexte trouvé par Coty pour exprimer son opposition à la politique de rapprochement avec l'Allemagne, au moment même où cette politique trouve sa première concrétisation décisive. Les accords de Locarno, par lesquels l'Allemagne reconnaît ses frontières occidentales établies par le traité de Versailles et qui constituent donc une reconnaissance implicite dudit traité, sont en effet, au moment où Coty publie sa tribune, sur le point d'être signés ${ }^{11}$. Or, à l'unisson de l'extrême droite et d'une bonne partie de la droite française, Coty est profondément hostile à ce rapprochement, et partisan de la poursuite de la politique de fermeté à l'égard de l'Allemagne. Cette fermeté ayant valu à la 
France son discrédit auprès de ses anciens alliés, Coty redoute la diffusion d'un film qui viendrait ajouter de l'eau au moulin de ses détracteurs par la mise en scène de l'attachement à une gloire nationale qui s'est traduite par la domination française sur l'Europe. La virulence de son propos s'explique d'ailleurs probablement par le fait qu'à l'automne 1925, pour les tenants de cette fermeté, la partie semble déjà perdue.

Gance répond dès le lendemain à Coty en lui opposant le pacifisme qui inspire ce film comme son œuvre tout entière, et en lui reprochant de jeter sur cette entreprise un opprobre qui pourrait décourager $\mathrm{d}^{\prime}$ éventuels repreneurs français ${ }^{12}$. Coty fait paraître le droit de réponse de Gance une semaine plus tard, non, cependant, sans le faire suivre d'une réponse de sa part, par laquelle il campe ostensiblement sur ses positions ${ }^{13}$. Il insiste sur le danger que représente à ses yeux la glorification de l'épopée napoléonienne et sur la crainte d'une possible exploitation de cette imagerie guerrière par les ennemis de la France, cette crainte étant en fait un prétexte pour donner une publicité à ses thèses nationalistes et complotistes. L'affaire Coty de septembre viendrait ainsi tout à la fois contredire et confirmer le malentendu soulevé par la «petite histoire» corse d'avril: ce film, qui pouvait aisément s'offrir à une appropriation fasciste, est finalement rejeté à Paris par celui-là même qui avait tenté de l'instrumentaliser à Ajaccio; mais, dans un cas comme dans l'autre, c'est bien d'opportunisme qu'il s'agit, et il apparaît que le réalisateur, dans les deux cas, a résisté à cette instrumentalisation.

Pourtant, les preuves existent que Gance a sciemment joué avec cette appropriation. Trois ans plus tard, au printemps 1928, il est en effet averti que la censure fasciste italienne impose, avant la première romaine du film, la suppression de plusieurs séquences, dont celle où La Marseillaise est chantée aux Cordeliers et l'ensemble de l'épisode $\operatorname{corse}^{14}$. Si la seconde coupure touche à l'ambiguïté du sentiment national italien à l'égard de la Corse et de Bonaparte, dont l'attachement à la France est probablement trop mis en évidence dans le film pour être acceptable par le nationalisme fasciste, la suppression de la scène de la présentation de La Marseillaise est indubitablement politique: l'exaltation de l'amour de la liberté et de l'enthousiasme révolutionnaire, même s'ils servent, dans le film, de terreau à l'avènement du chef qui parviendra à les dompter, est évidemment inacceptable pour un régime qui s'est établi sur la répression des velléités révolutionnaires et sur la négation des droits humains fondamentaux hérités de la Révolution française. Pourtant, l'attachement viscéral de Gance à son œuvre le pousse à passer outre cette logique pour solliciter la bienveillance personnelle du Duce, se mettant ainsi en position de susciter une réappropriation explicitement fasciste, cette fois-ci, de son film.

C'est ce qui ressort de la lettre qu'il adresse à Mussolini lui-même, par laquelle, avant de terminer sur «la profonde assurance de [son] admiratif et très respectueux dévouement», il implore le Duce:

Je viens vous supplier, Excellence, de bien vouloir prendre quelques instants [sic] pour la projection de mon œuvre que l'Ambassadeur de France à Rome vous montrera. Je veux penser que cet incident se tournera à mon avantage en 
prouvant à Son Excellence que quelques œuvres françaises travaillent en silence dans votre esprit pour le monde entier. ${ }^{15}$

Même si Gance n'a ici pour but que de flatter son interlocuteur afin d'obtenir l'annulation de la censure de son film, les mots qu'il emploie pour ce faire sont lourds de sens: ils signalent sans ambiguïté que Gance lui-même voit dans ce film l'instrument possible d'une propagande en faveur de $l^{\prime}$ «esprit» fasciste - le culte de l'ordre et de l'autorité du chef - dans «le monde entier».
À la lumière de la «petite histoire» corse et de ses prolongements, la violente critique de Moussinac renvoie brutalement le réalisateur à sa responsabilité publique, lui qui, tout en clamant l'apolitisme de son cuvre, n'a eu de cesse, volontairement ou non, de l'exposer à sa politisation.

Dans le contexte de la France des années vingt, cette évocation cinématographique de l'épopée napoléonienne se révèle propre à réveiller les démons et à susciter les passions que Gance se donnait précisément pour but de transcender.

\section{Notes}

1 Léon Moussinac, «Un film français: Napoléon», L'Humanité, 23 avr. 1927.

2 Ibid. Souligné par l'auteur.

3 C'est le 22 avril 1927 que Sarraut prononce son discours de Constantine, où il s'écrie, paraphrasant, en la détournant, la célèbre invective de Clemenceau contre le cléricalisme: «Le communisme, voilà l'ennemi! » Voir Serge Berstein, Jean-Jacques BecKer, Histoire de l'anticommunisme en France, O. Orban, t. 1, 19171940, 1987, p. 188-190, 213-214.

4 François Coty a pris la tête du Figaro en 1922, avant de racheter Le Gaulois, puis Le Charivari, de financer temporairement L'Action française et finalement de lancer, en 1928, le quotidien populiste, xénophobe et antisémite $L^{\prime} A m i d u$

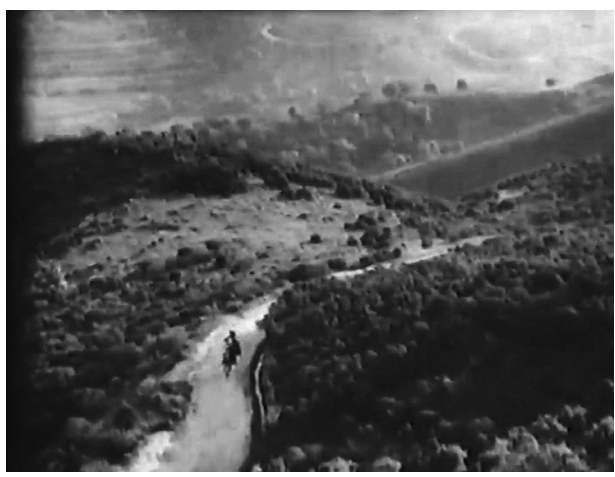

Bonaparte (Albert Dieudonné) galopant sur les routes de Corse

Peuple. Par ailleurs, il est un des principaux soutiens financiers, dans la seconde moitié des années vingt, du Faisceau de Georges Valois et de l'Action française, avant de contribuer, en 1927, à la création des Croix-de-Feu. Voir Fred Kupferman, "François Coty», dans Oliver BArrot, Pascal Ory (dir.), Entre deux guerres. La création française (1919-1939), Fr. Bourin, 1990, p. 77-92.

5 Pierre ScIze, «On "tourne" au pays de Napoléon ", Lectures pour tous, août 1928.

6 Voir Kevin Brownlow, Napoléon. Le grand classique d'Abel Gance, traduit de l'anglais [2004] par Christine Leteux, A. Colin, 2012, p. 9293. L'événement est même à peine évoqué par Jean-Pierre Mattei dans le catalogue de l'exposition qui s'est tenue du 31 mars au 
3 juillet 2016 à Ajaccio, au Musée national de la Maison Bonaparte (Jean-Pierre MATteI, Napoléon vu par Abel Gance. La séquence corse, Albiana, 2016, p. 22).

7 Kevin Brownlow, loc. cit.

8 Devenu par ailleurs membre des Croix-de-Feu, Paoli fait ainsi acclamer le 9 mai 1936, en conseil municipal, la conquête de l'Éthiopie par l'armée mussolinienne. En 1940, le régime de Vichy le confirme dans ses fonctions, dont il sera démis par la Résistance corse, qui le contraindra à l'exil en Algérie, puis à Nice. Voir Paul Silvani, Le Bonapartisme. Une saga corse, Albiana, 2003, p. 232 sq.

9 François CотY, «Pourquoi je ne donne pas mon concours au film dont Napoléon sera l'étoile», Le Figaro, 28 sept. 1925.
10 Ibid.

11 Les accords de Locarno seront signés le 16 octobre 1925, et seront suivis en 1926 par l'entrée de l'Allemagne à la SDN.

12 Lettre de Gance à François Coty, 30 sept. 1925. CF, fonds Abel Gance, 171-B56.

13 "Le film "Napoléon" » [droit de réponse d'Abel Gance et réponse de François Coty], Le Figaro, 6 oct. 1925.

14 Voir la lettre de Gance à Gabriele D'Annunzio, 29 mars 1928. CF, fonds Abel Gance, 179-B61.

15 Lettre de Gance à Benito Mussolini, 29 mars 1928. CF, fonds Abel Gance, 179-B61. 